\title{
Effectiveness of Strengths-based Case Management for People with Mental Health Problems in Hong Kong
}

\author{
Kevin Y. C. Hui \\ Cressida W. C. Leung \\ Morgan C. K. Ng \\ Wing Ching Yu \\ Edison K. L. Lau \\ Siu-kau Cheung
}

\begin{abstract}
This study examined the effectiveness of a 6-month strengths-based case management intervention with 45 Chinese participants with mental health problems in Hong Kong. Social workers provided service according to the strengths-based case management (SCM) model developed at the University of Kansas. Changes in participants' recovery components (Stage of Recovery Scale), mental health symptoms (GHQ), and satisfaction with life were assessed using a single group pretest and posttest design. Results suggest that participants had some improvement in their autonomy, hope, and overall wellbeing as well as satisfaction with life after receiving services. No significant improvements in the other recovery components and GHQ score were found. Significantly, a number of participants progressed from stages of being overwhelmed or struggling with disability to stages of living with or beyond disability. Strengths-based practice helped participants develop a transformed self which sees hope and possibility despite the vulnerabilities caused by their illness. Though further refinement and testing are vital, adoption of SCM in Hong Kong mental health services is promising.
\end{abstract}

Keywords: Strengths-based practice, recovery, mental health, Hong Kong

Mental health problems have become a major concern in Hong Kong. As the population's awareness of mental health issues heightens, the demand for mental health service has rapidly increased. In recent years more and more resources have been allocated to Hong Kong mental health settings. To cope with this high demand and the associated costs, a more effective and efficient model for mental health service is needed. Traditionally, psychiatric services in Hong Kong have followed the medical model with psychiatric drugs being the major treatment option for people with psychosocial disabilities (Lee \& Yiu, 2013; Siu \& Sung, 2013). The emphasis has been on treating symptoms and/or pathologies based on psychiatric diagnoses, with the aim of restoring individuals to the level of functioning before their mental illness surfaced.

In the last two decades, growing evidence suggests that the medical model is not as effective as practitioners had once thought in either lessening the incidence and prevalence of various mental health disorders or promoting psychosocial betterment in our world (Hillman \& Ventura, 1992). Some findings even indicated that the medical model could have negative impacts on service recipients (e.g., low self-esteem; see Xie, 2013 for a brief review). These observations triggered the development of an alternative approach in the early 1980's, namely, the strengths-based approach (Rapp \& Sullivan, 2014). This approach provides practitioners with a new perspective of mental health service which allows them to direct the service recipients to a new recovery journey that is full of possibilities, options, and wellness (Rapp \& Goscha, 2011).

Kevin Y. C. Hui (kevinhui@sracp.org.hk), Cressida W. C. Leung (m_vp@sracp.org.hk), Morgan C. K. Ng, Wing-ching Yu, and Edison K. L. Lau (rdo@sracp.org.hk) are with The Society of Rehabilitation and Crime Prevention, Hong Kong, Unit 11B, 9/F, Tower B, Hunghom Commercial Centre, 37 Ma Tau Wai Road, Hunghom, Kowloon, Hong Kong. Siu-kau Cheung (s.k.cheung@cityu.edu.hk), is with Community College of City University of Hong Kong Division of Social Sciences, 88 Tat Chee Avenue, City University of Hong Kong, Kowloon Tong, Hong Kong. 
Although strengths-based social work practice has gained popularity in the west, it is still in its infancy in mental health settings in Hong Kong. Until the early 2010s, social workers mainly followed the mental rehabilitation model and focused on alleviation of psychiatric symptoms and restoration of adaptive functions of people with mental illness (Liu, 2014). There were applications of the strengths perspective to individual cases with dementia and self-cutting behaviors (Yip, 2005, 2006), but systematic applications to a wider population were scant. In 2010, the Social Welfare Department of the Hong Kong government established Integrated Community Centers for Mental Wellness (ICCMW) in all districts across the territory. As their name suggests, the centers take a more positive focus aimed at enhancing the social support and re-integration of individuals with mental illness into the community (Lee \& Yiu, 2013). These centers provide one-stop, districtbased, and accessible community support and social services ranging from early prevention to risk management for service users. Only then did the concept of strengths-based practice begin to take root in the social work field in the city.

The strengths-based case management (SCM) intervention examined in this study was a project undertaken in two ICCMWs in Hong Kong for a sample of people with mental illness. Using a single group pretest and posttest design, the study aimed to examine the applicability and effectiveness of the SCM model in a Chinese population.

\section{The Concept of Recovery}

An associated development in psychiatric rehabilitation in recent decades is the notion of recovery. Recovery has become an ultimate goal of treatment for people with chronic mental illness in western societies (Song \& Hsu, 2011). Despite the absence of a universal definition of recovery, the definition proposed by Deegan (1988) has been generally accepted.

Recovery is a process, a way of life, an attitude, and a way of approaching the days' challenges. It is not a perfectly linear process. At times our course is erratic, we falter, slide back, regroup and start again... The need is to meet the challenge of disability and to re-establish a new and valued sense of integrity and purpose within and beyond the limits of the disability; the aspiration is to live, work and love in a community in which one makes a significant contribution. (p. 15)

According to this definition, recovery is a comprehensive concept encompassing both process and outcome. It is about an attitude as well as a way of life. In this sense, there is never an endpoint in recovery. A "recovering” person is not one who will no longer struggle and experience symptoms, never use mental health services and medications again, or is completely independent in his/her life (Rapp, 1998). Rather, the person has come to accept his/her symptoms and struggles, think forward with hope, and take proactive control in his/her life.

Various researchers have proposed components that may foster recovery in people with mental illness, including the emergence of hope, taking responsibility for one's own wellness, effective management of one's own disability, developing a self-identity with potential, developing new meaning and a sense of purpose in life, advocacy for one's own rights, and peer support (Lee \& Yiu, 2013; Mead \& Copeland, 2005). Based on the recovery components, Song and Hsu (2011) charted the recovery process in terms of four stages: (1) overwhelmed by the disability, (2) struggling with disability, (3) living with disability, and (4) living beyond disability. The stages represent progressive development towards recovery, with the higher stages characterized by an increase in the process components of regaining autonomy, disability management/taking responsibility and sense of hope, and the positive outcomes of improving social functioning/role performance, overall well- 
being, and willingness to help. Song and Hsu (2011) developed the Stages of Recovery Scale and argued that it could be used for assessing the status of service users in both recovery components and outcomes.

\section{Strengths-Based Approach}

As recovery focuses on valuing and building one's inherent worth, resilience, and capacities, treatment methods that merely alleviate symptoms of the disorder have become insufficient (Hillman \& Ventura, 1992). In this regard, the strengths-based approach appears to be a promising alternative (Xie, 2013).

The strengths-based approach is a hope-inducing and energizing approach which emphasizes possibilities, options, and wellness rather than problems, constraints and sickness (Rapp \& Goscha, 2011). Its focus is not only on the intrapersonal and interpersonal qualities of a person but also on the resources in their environment (Saleebey, 2006). Different strengths-based practices have been developed, including the strengths-based approach (Rapp, 1994), the asset-building model of community development (Kretzman \& McKnight, 1993), individual placement and support model of supported employment (Becker \& Drake, 2003), and solution-focused brief therapy (Miller, Hubble, \& Duncan, 1996). The strengths model proposed by Rapp embodies six vital principles: (1) a person has the capacity to recover, reclaim and transform; (2) the focus is on human strengths rather than weakness/deficits; (3) mental health services are directed by the service recipients; (4) the therapeutic relationship is primary and essential; and (5) community is indeed the preferred setting to work in because (6) it is the oasis of resources.

Following these six principles, Rapp (1998) designed the SCM model, an intervention that is both a philosophy of practice and a set of tools. The SCM aims to enhance recovery through a client-directed and goal-oriented approach of service delivery. The model includes five main steps: engagement, assessment, case planning, resources acquisition, and tracking and evaluation (Rapp \& Goscha, 2011). After the initial engagement, practitioners implement strengths assessment to guide the service recipients to identify their strengths, personal medicines (the things that people do to create wellness and betterment in their lives), environmental resources, and personal goals. Based on these elements, a personal recovery plan is then collaboratively compiled. Following the plan, service recipients are assisted to acquire the available resources and to achieve their set goals. Throughout the process, tracking and evaluation are done periodically to ensure the applicability of the service recipients' plans in different periods and to credit them in order to encourage and support them to move forward on their recovery journeys. Even though problems, constraints and sickness are not the main concern of SCM, they are addressed in the context of goal attainment in the service recipients' recovery journey (Fukui et al., 2012).

\section{Effectiveness of Strengths-Based Intervention}

Empirical research has suggested that SCM has a positive impact on one's well-being as well as symptoms management. Macias, Kinney, Farley, Jackson and Vos (1994) found that consumers with psychiatric symptoms who received SCM in conjunction with psychosocial rehabilitation showed statistically significant better physical and mental health than those who received only psychosocial rehabilitation. They reported less mood disturbance, fewer maladaptive thoughts but clearer and more rational thoughts in comparison to a control group. In a later quasi-experimental study, Macias, Farley, Jackson, and Kinney (1997) found that SCM was effective in helping adults with serious mental illness to achieve community support goals and reduced symptomatology. Similar results were found in Barry, Zeber, Blow, and Valenstein's (2003) study. Smock et al. (2008) 
argued that it is the strengths identification and utilization as well as hope induction and maintenance that lead to improvement in one's well-being and symptoms management capability.

Other studies have suggested that people receiving SCM were more independent in their daily living. They were found to lead more purposive lives (Kisthardt, 1993) and possess more effective community living skills (Stanard, 1999). Also, SCM intervention appeared to enhance positive employment outcomes (Siegal et al., 1996) and facilitate proactive use of leisure time (Modrcin, Rapp, \& Poertner, 1988).

Despite some evidence about the positive outcomes of strengths-based practice, relevant research to date is still far from adequate and conclusive. Recent reports of its effectiveness are scant. Rapp and Sullivan (2014) maintained that there is a need for rigorous research to examine the effectiveness of the model with different client groups and that adopt pre-post designs as a precursor to experimental testing.

In Hong Kong, as the broad concept of recovery has gained acceptance in mental health services, there were attempts to test the effectiveness of a number of recovery-oriented pilot programs, including Wellness Recovery Action Planning (WRAP; Mak, 2013) and the Peer Support Worker Project (Yeung et al., 2013). These were mainly project-based interventions; none related to SCM have been examined. The current project was the first to investigate the effectiveness of SCM intervention on the recovery of people with mental illness in Hong Kong.

\section{Present Study Design and Hypothesis}

A single group pretest and posttest design was used in the current study. The mental health practitioners (23 social workers) had both the philosophy as well as the tools - the strengths assessment form and the personal recovery plan - of SCM proposed by Rapp (1994). These social workers received more than 30 hours in SCM training and supervision by experienced SCM practitioners from the University of Kansas through Skype. They were supervised by four senior local social workers (the supervisors) who had received onsite SCM training at the University of Kansas and Johnson County Mental Health Center. The social workers provided a six-month SCM intervention for a group of adults with a diagnosis of mental illness. To maintain the fidelity of SCM, the social workers wrote detailed case recordings after every meeting with the participants. Throughout the intervention period, each social worker attended twelve group supervision sessions conducted by the senior social workers, once every two weeks. In each session, the case recordings as well as the strengths-based assessments were discussed.

During the intervention, the worker used the Strengths Assessment Form (Rapp \& Goscha, 2011) to help participants visualize their personal and environmental resources and identify the strengths they currently possessed, accumulated, or made use of in the past. Typical of Chinese people, the participants tended to be overly humble and discount their strengths, achievements, and resources. The worker had to be patient and persistent to help the participants see the values and virtues of their assets, no matter how small and mundane they seemed. Participants' goals and aspirations were also recorded in the Strengths Assessment Form. The goals were broken down into small, measurable, and attainable steps and put into the Personal Recovery Plan. Progress toward goal accomplishment was reviewed every four to six weeks, and the recovery plans were updated if necessary. To recognize members' specific achievements, celebrations in the form of a lunch or a certificate were organized. In whatever form it might take, modesty was practiced because in Chinese culture ostentation might create embarrassment for those who considered themselves unworthy of the kudos (Bond, Lun, Chan, Chan, \& Wong, 2012). 
The effectiveness of the intervention was evaluated through examining the participants' change in the components of recovery, mental health status, and life satisfaction after receiving the service. It was hypothesized that SCM would facilitate the process and outcome of recovery of participants, which in turn would lead to better overall well-being as well as higher life satisfaction.

\section{Methods}

\section{Participants}

Participants were recruited from two ICCMWs operated by the same organization in Hong Kong. Though situated in two different districts (both working to middle class), the nature of the services provided were largely the same. All service users seeking counseling service in the period from February 2014 to January 2015 were invited to participate in the study, and they joined on a voluntary basis with no incentive given. The participants were all Chinese-speaking adults diagnosed with a mental illness without immediate suicide and/or risk of violence. People suffering from intellectual disability, dementia, substance abuse, personality disorder, and/or autism were excluded. A total of 67 participants were recruited at the baseline and 45 completed the SCM intervention. Twenty-two participants dropped out due to moving out of the service district or they found filling out the questionnaires too time-consuming. The analyses in this study were based on the 45 complete sets of data. Independent samples $t$-tests and cross- tabulations were conducted to examine whether the 22 participants who dropped out differed from the 45 participants who completed the study in terms of demographic characteristics and the dependent variables. No significant differences between the two groups were found.

Among the 45 participants, 19 were men and 26 were women. Their ages ranged from 18 to $74(M=36.4, S D=13.38)$. They were born either in Hong Kong or mainland China, and were all currently living in Hong Kong. Most of the participants (64.4\%) were living with family or friends, $26.7 \%$ were living alone, and $8.9 \%$ were living in private hostels providing mainly residential service. The majority $(n=25,55.6 \%)$ had secondary school education, $24.4 \%$ had primary school education or below, and $20 \%$ had a college education or above.

The predominant diagnosis of the participants was mood disorder ( $n=25,55.6 \%$ ), followed by schizophrenia and other psychotic disorders ( $n=17,37.8 \%)$, adjustment disorder ( $n=2,4.4 \%)$, and sleeping disorder $(n=1,2.2 \%)$. The mean age of onset was 36.4 $(S D=15.27)$. Twenty-seven had been hospitalized with the mean number of hospitalizations since onset being 2.9 times ( $S D=2.39$ ). The rest reported no hospitalization experience.

\section{Measures}

Three self-reported questionnaires were used in the current study: the Stages of Recovery Scale (SRS; Song \& Hsu, 2011), the Chinese version of the General Health Questionnaire (C-GHQ-12; Lee et al., 1985), and the Chinese version of the Satisfaction with Life Scale (SWLS; Diener, Emmons, Larsen, \& Griffin, 1985). These three scales formed the pre and post-assessment batteries.

Stages of Recovery Scale (SRS). SRS is a self-reported, Chinese scale to measure the personal recovery of people with mental illness (Song \& Hsu, 2011). It comprises six subscales with a total of 45 items. Three of the subscales measure the process of recovery (regaining autonomy, disability management/taking responsibility, and sense of hope) and the other three measure the outcome of recovery (social functioning/role performance, overall well-being, and willingness to help). Participants were asked to indicate the 
frequency of occurrence of the situations described by the items using a 4-point Likert scale, ranging from 0 (never) to 3 (often). The total score of these six subscales were used to define the stage of recovery following the cut-off points suggested by Song and Hsu (2011): Stage 1-Over-whelmed by the disability (SRS score 0-57), Stage 2-Struggling with the disability (SRS score 58-90), Stage 3-Living with the disability (SRS score 91-119), and Stage 4-Living beyond the disability (SRS score 120-135). The SRS has been shown to demonstrate good psychometric properties, including test - retest reliability and internal consistency for the overall scale and the subscales. The current study yielded a Cronbach's alpha of .96 for the overall scale score and .66 to .94 for the subscale scores.

The Chinese Version of the General Health Questionnaire-12 (C-GHQ-12). The short form of the Chinese version of the General Health Questionnaire is a self-reported scale commonly used to measure mental health status and distress in a Hong Kong population (Goldberg \& Williams, 1988; Lee, Lam, Ong, Wang, \& Kleevens, 1985). It consists of 12 items (six positive and six negative) measuring the frequency of nonpsychotic mental health symptoms on a 4-point scale ranging from 1 (never) to 4 (usually). Examples of the positive items include "able to concentrate" and "playing a useful part in things," whereas negative items include "loss of sleep over worry" and "felt constantly under strain.” The positive items were corrected from 1 (usually) to 4 (never) and summed with the negative ones. High scores indicate worse health. The C-GHQ-12 was found to correlate strongly with concurrently reported symptoms of physical symptoms of pain and anxiety-related somatic symptoms (Lee et al., 1985). High reliability and internal consistency of the scale scores have been obtained. The current study yielded a Cronbach's alpha of .92.

The Chinese Version of the Satisfaction with Life Scale (C-SWLS). The Satisfaction with Life Scale is a validated self-reported scale used to measure global life satisfaction (Diener et al., 1985). Participants indicated the extent to which they agreed or disagreed with five items using a 7-point Likert scale ranging from 1 (strongly disagree) to 7 (strongly agree). Examples of the items include "In most ways my life is close to my ideal," "The conditions of my life are excellent," and "I am satisfied with my life." Confirmatory factor analysis of the scale showed support for a unidimensional factor structure of general overall life satisfaction (Sachs, 2004). C-SWLS has been found to have good internal consistency across studies (Pavot \& Diener, 1993). The current study yielded a Cronbach's alpha of .89 .

\section{Procedures}

The Research Monitoring Committee of the organization operating the two ICCMWs conducted an ethical review of the study and granted approval to proceed with the data collection in the centers. Prior to the beginning of the intervention, an intake interview was conducted in which the nature of the study was explained. Informed consent was obtained from the participants before they were requested to fill in the pre-battery assessments.

Participants received a six-month intervention by a social worker who practiced according to the SCM model, while they maintained their regular psychiatric follow-up. During the intervention, participants had an average of 5.4 face-to-face contacts (range from 4 to 7 times) with the social worker, each lasting a minimum of 60 minutes. At the completion of the intervention, participants filled out the post-intervention batteries and were debriefed.

In order to examine the change in the process and outcome variables after the sixmonth intervention, seven paired sample $t$-tests were conducted to analyze the differences between the pre and post-intervention scores of the SRS as well as its six subscales, the C- 
GHQ-12, and the C-SWLS. In order to understand whether there was an interaction between the intervention and the participants' background factors on the dependent variables, repeated measures analyses of variance were conducted on SRS, C-GHQ-12, and the C-SWLS, using gender (male vs. female), diagnosis (affective disorder vs. psychotic related disorder; adjustment disorder and sleeping disorder were excluded from this analysis), living status (alone vs. in an institution vs. with family/friends) and hospitalization (none vs. more than one) as covariates. These variables were chosen as covariates because they were found to correlate with some of the SRS scale scores (see Table 1 and 2 for correlation matrices of all variables). A chi-square analysis was performed on the change of recovery stage.

\section{Results}

\section{Stage of Recovery Scale (SRS)}

Overall score of SRS. Results of a paired sample $t$-test performed on the pre and postintervention scores of SRS revealed a statistically significant difference, $t_{(44)}=-2.60, p<.05$, $d=.36$, in which the average post-intervention score of SRS $(M=95.9)$ was higher than the average pre-intervention score $(M=87.1)$ (see Table 3 ). This result suggested the participants had an improvement in the recovery processes and outcomes of small to medium practical significance after receiving the SCM intervention.

The results of the repeated measures ANOVA revealed no statistically significant interaction effects between the pre and post-intervention scores of the SRS and gender (male vs. female; $F_{(1,43)}=0.41, p=.53$ ), diagnosis (affective disorder vs. psychotic related disorder; $F_{(1,40)}=0.02, p=.89$ ), living status (alone vs. in an institution vs. with family/friends; $F_{(1,43)}=1.96, p=.17$ ) or hospitalization (none vs. once or more than; $F_{(1 \text {, }}$ ${ }_{41)}=0.15, p=.70$ ). These indicated that the inter-individual characteristics (i.e., gender, diagnosis, living status and hospitalization) had no statistically significant interaction effects on one's recovery due to the SCM intervention. 
Table 1. Correlations Between the Pre-Intervention Dependent Variables and Four Covariates ( $n=45)$

\begin{tabular}{|c|c|c|c|c|c|c|c|c|c|c|c|c|}
\hline & 1 & 2 & 3 & 4 & 5 & 6 & 7 & 8 & 9 & 10 & 11 & 12 \\
\hline 1. Gender & & & & & & & & & & & & \\
\hline 2. Diagnosis & .04 & & & & & & & & & & & \\
\hline 3. Hospitalization & -.28 & $-.47 * *$ & & & & & & & & & & \\
\hline 4. Living Status & .06 & -.18 & .01 & & & & & & & & & \\
\hline 5. Pre-SRS Overall score & .00 & -.28 & .21 & .05 & & & & & & & & \\
\hline 6. Pre-SRS Regaining Autonomy & -.03 & -.22 & .20 & -.02 & $.93 * *$ & & & & & & & \\
\hline $\begin{array}{l}\text { 7. Pre-SRS Disability Management/ } \\
\text { Taking Responsibility }\end{array}$ & -.02 & -.16 & -.06 & -.07 & $.78^{* *}$ & $.69 * *$ & & & & & & \\
\hline 8. $\quad$ Pre-SRS Overall Well-being & -.01 & -.32 & $.32 *$ & .04 & $.83^{* *}$ & $.68 * *$ & $.46^{* *}$ & & & & & \\
\hline $\begin{array}{l}\text { 9. Pre-SRS Social Functioning/Role } \\
\text { Performance }\end{array}$ & .05 & -.20 & .25 & .28 & $.85^{* *}$ & $.68^{* *}$ & $.58 * *$ & $.68 * *$ & & & & \\
\hline 10. Pre-SRS Sense of Hope & .19 & -.26 & .01 & .14 & $.46^{* *}$ & $.45 * *$ & .17 & $.35^{*}$ & $.40 * *$ & & & \\
\hline 11. Pre-SRS Helping Others & -.05 & -.22 & .20 & .05 & $.82 * *$ & $.70 * *$ & $.62 * *$ & $.64 * *$ & $.81 * *$ & .19 & & \\
\hline 12. Pre-GHQ & -.06 & .22 & $-.41 *$ & -.08 & -.30 & -.21 & -.01 & $-.55^{* *}$ & -.19 & -.19 & -.28 & \\
\hline 13. Pre-SWLS & -.14 & -.13 & $.33^{*}$ & .17 & $.51 * *$ & $.45 * *$ & .12 & $.71^{* *}$ & $.46^{* *}$ & .22 & .37 & -.47 \\
\hline
\end{tabular}

Note. SRS=Stage of Recovery Scale; GHQ=General Health Questionnaire; SWLS=Satisfaction with Life Scale. ${ }^{*} p<.05 ; * * p<.01$ 
Table 2. Correlations Between the Post-Intervention Dependent Variables and Four Covariates ( $n=45)$

\begin{tabular}{|c|c|c|c|c|c|c|c|c|c|c|c|c|}
\hline & 1 & 2 & 3 & 4 & 5 & 6 & 7 & 8 & 9 & 10 & 11 & 12 \\
\hline \multicolumn{13}{|l|}{ 1. Gender } \\
\hline 2. Diagnosis & .04 & & & & & & & & & & & \\
\hline 3. Hospitalization & -.28 & -.47 & & & & & & & & & & \\
\hline 4. Living Status & .06 & -.18 & .01 & & & & & & & & & \\
\hline 5. Post-SRS Overall score & .09 & -.27 & .16 & .24 & & & & & & & & \\
\hline 6. Post-SRS Regaining Autonomy & .15 & -.28 & .13 & .26 & $.97 * *$ & & & & & & & \\
\hline $\begin{array}{l}\text { 7. Post-SRS Disability Management / } \\
\text { Taking Responsibility }\end{array}$ & -.01 & -.09 & .05 & .14 & $.90 * *$ & $.85^{* *}$ & & & & & & \\
\hline 8. Post-SRS Overall Well-being & .06 & $-.34 *$ & $.32 *$ & .15 & $.85^{* *}$ & $.75^{* *}$ & $.70 * *$ & & & & & \\
\hline $\begin{array}{l}\text { 9. Post-SRS Social Functioning/Role } \\
\text { Performance }\end{array}$ & .07 & -.30 & .16 & .24 & $.92^{* *}$ & $.97^{* *}$ & $.79 * *$ & $.76^{* *}$ & & & & \\
\hline 10.Post-SRS Sense of Hope & .12 & -.18 & .05 & .24 & $.81 * *$ & $.81^{* *}$ & $.73^{* *}$ & $.59 * *$ & $.66^{* *}$ & & & \\
\hline 11.Post-SRS Helping Others & .09 & -.21 & .03 & .29 & $.93^{* *}$ & $.84^{* *}$ & $.67^{* *}$ & $.59 * *$ & $.77 * *$ & $.74^{* *}$ & & \\
\hline 12.Post-GHQ & -.27 & .34 & -.24 & -.13 & $-.65 * *$ & $-.62 * *$ & $-.45^{* *}$ & $-.73 * *$ & $-.53 * *$ & $-.58 * *$ & $-.53 * *$ & \\
\hline 13.Post-SWLS & .12 & -.38 & $.40^{* *}$ & .21 & $.59 * *$ & $.55^{* *}$ & $.46^{* *}$ & $.73^{* *}$ & $.50 * *$ & $.48^{* *}$ & $.30^{*}$ & $-.58 * *$ \\
\hline
\end{tabular}

Note. SRS=Stage of Recovery Scale; GHQ=General Health Questionnaire; SWLS=Satisfaction with Life Scale. ${ }^{*} p<.05 ; * * p<.01$ 
Table 3. Paired Sample t-test Results for Measures of SRS, GHQ and SWLS (N=45)

\begin{tabular}{|c|c|c|c|c|c|c|}
\hline \multirow[b]{2}{*}{ Scales } & \multicolumn{2}{|c|}{ Pre-Intervention } & \multicolumn{2}{|c|}{$\begin{array}{c}\text { Post- } \\
\text { Intervention }\end{array}$} & \multirow[b]{2}{*}{$t$} & \multirow[b]{2}{*}{$p$} \\
\hline & $M$ & $S D$ & $M$ & $S D$ & & \\
\hline \multicolumn{7}{|l|}{ Stage of Recovery Scale } \\
\hline Overall Score & 87.1 & 23.45 & 95.9 & 25.29 & -2.60 & $.01 *$ \\
\hline \multicolumn{7}{|l|}{ Process variables } \\
\hline Regaining Autonomy & 29.5 & 8.93 & 32.9 & 9.41 & -2.44 & $.02 *$ \\
\hline Sense of Hope & 6.3 & 1.80 & 6.9 & 1.76 & -2.37 & $.02 *$ \\
\hline $\begin{array}{l}\text { Disability Management/ } \\
\text { Taking Responsibility }\end{array}$ & 18.9 & 5.13 & 20.3 & 5.14 & -1.79 & .08 \\
\hline \multicolumn{7}{|l|}{ Outcome variables } \\
\hline Overall Well-being & 13.4 & 6.07 & 15.4 & 5.50 & -2.54 & $.02 *$ \\
\hline $\begin{array}{l}\text { Social Functioning/Role } \\
\text { Performance }\end{array}$ & 12.6 & 4.10 & 13.7 & 4.08 & -1.77 & .08 \\
\hline Helping Others & 6.4 & 2.28 & 6.7 & 2.00 & -0.95 & .35 \\
\hline General Health Questionnaire & 17.4 & 7.39 & 16.1 & 6.13 & 1.14 & .26 \\
\hline Satisfaction with Life Scale & 19.6 & 7.37 & 22.0 & 6.85 & -2.50 & $.02 *$ \\
\hline
\end{tabular}
Note. ${ }^{*} p<.05$.

Recovery stage. Participants' recovery stages were defined following the cut-off points suggested by Song and Hsu (2011). A chi-square analysis was performed to analyze the change in the recovery stage of participants before and after receiving strength-based intervention. Results revealed that there was a statistically significant difference between the pre and post recovery stages of participants, $\chi_{(1, n=45)}^{2}=6.79, p<.01$. Nine of the 45 participants progressed to a higher stage as a result of the intervention, but five regressed to a lower stage (see Table 4). Most of the participants (68.9\%) remained in the same stage.

Table 4. Chi-square Test Result for Stage of Recovery by Period

\begin{tabular}{|c|c|c|c|c|}
\hline \multirow[b]{2}{*}{ Post-Stage of Recovery } & \multicolumn{2}{|c|}{ Pre-Stage of Recovery } & \multirow[b]{2}{*}{$\chi^{2}$} & \multirow[b]{2}{*}{$p$} \\
\hline & $\begin{array}{l}\text { Overwhelmed/ } \\
\text { Struggling With Disability }\end{array}$ & $\begin{array}{l}\text { Living With or } \\
\text { Beyond Disability }\end{array}$ & & \\
\hline \multirow{3}{*}{$\begin{array}{l}\text { Overwhelmed/ } \\
\text { Struggling With Disability } \\
\text { Living With or Beyond } \\
\text { Disability }\end{array}$} & $16(35.56 \%)$ & $5(11.11 \%)$ & \multirow{3}{*}{6.79} & \multirow{3}{*}{$.01^{* *}$} \\
\hline & & & & \\
\hline & $9(20 \%)$ & 15 (33.33\%) & & \\
\hline
\end{tabular}

Note. ${ }^{* *} p<.01$

\section{General Health Questionnaire (GHQ)}

A paired sample $t$-test was conducted to analyze the difference between the pre and post-intervention scores of the GHQ. The difference was not statistically significant, $t_{(36)}=1.14, p=.26$ (see Table 3). Similarly, repeated measures ANOVAs were conducted to analyze the differences between the pre and post-intervention scores of the GHQ with gender (male vs. female), diagnosis (affective disorder vs. psychotic related disorder; adjustment disorder and sleeping disorder were excluded from this analysis), living status (alone vs. in an institution vs. with family/friends) and hospitalization (none vs. more than one) as covariates (see Table 1 and 2 for correlation). No statistically significant interaction effects between the pre and post-intervention scores of GHQ and gender, $F_{(1,35)}=1.08$, $p=.31$, diagnosis, $F_{(1,33)}=.54, p=.47$, living status, $F_{(1,35)}=.12, p=.74$, and hospitalization, 
$F_{(1,33)}=4.03, p=.053$, were found.

\section{Satisfaction with Life Scale}

A paired sample $t$-test was conducted to analyze the difference between the pre and post-intervention scores of the SWLS. Results revealed that there was a statistically significant difference between the pre and post-intervention scores of the SWLS, $t_{(44)}=-$ 2.50, $p<.05, d=.34$. The average post-intervention score of SWLS $(M=22.0)$ was higher than the average pre-intervention score $(M=19.6)$ (see Table 3). Overall, participants were more satisfied with their life after receiving the SCM intervention, with an effect size of small to medium practical significance.

Similarly, repeated measures ANOVAs on the pre- and post-intervention SWLS scores using gender (male vs. female), diagnosis (affective disorder vs. psychotic related disorder; adjustment disorder and sleeping disorder were excluded from this analysis), living status (alone vs. an in institution vs. with family/friends) and hospitalization (none vs. more than one) as covariates (see Table 1 and 2 for correlation matrix) showed no statistically significant interaction effects between the pre and post-intervention scores of the SWLS in terms of gender, $F_{(1,43)}=3.49, p=.07$, diagnosis, $F_{(1,40)}=0.60, p=.44$, living status $F_{(1,}$ 43) $=0.03, p=.87$, and hospitalization, $F_{(1,41)}=0.10, p=.76$.

\section{Discussion}

The current study provides the first empirical evaluation of SCM practice with people with mental illness in a mental health setting in Hong Kong. Though the design lacks the rigor of randomized control group designs, it does provide data regarding the effectiveness of SCM in improving and enhancing the recovery of individuals with mental illness.

The findings of the current study produced mixed results. On the one hand, the SCM intervention in the study brought about significant though small improvements in mental health recovery. More promising changes were observed in two process components (autonomy and hope) and one outcome component (overall well-being) after the SCM intervention. Given the emphasis on goal orientation, strengths assessment and hope inducement of SCM, it is not surprising to note the positive outcomes regarding the two process components. Since autonomy and hope are associated with general well-being (Rapp \& Goscha, 2004), the enhancement of these elements likely contributed to the improvement in participants' overall well-being in the current study.

Nevertheless, no statistically significant improvement was found in the process component of disability management/taking responsibility and the outcome components of social functioning/role performance and helping others. The GHQ results also indicated that SCM failed to bring about reduction of psychological distress among the participants. Behind the apparently disappointing façade, the findings actually have important implications for the effectiveness of strengths-based practice. First, while the six-month intervention may facilitate the recovery process, these intermediate changes may not be translated immediately into enhanced personal and social functioning outcomes. Second, despite the persistence of certain psychological distress and symptoms as indicated by the GHQ, the individuals experienced an enhanced sense of overall well-being and satisfaction with life. These positive changes may have been downplayed if the focus remained on alleviation of symptoms, illness, and dysfunction. Actually, the experience of the participants in this study exemplifies the notion of recovery which "involves a transformation of the self-wherein one both accepts one's limitations and discovers a new world of possibility" (Deegan, 1988, p. 13). The participants in this study are not "cured" 
or free from mental health problems, but they can lead more satisfying, hopeful, and contributing lives despite their illness.

In terms of stage of recovery, some participants progressed into a higher stage but a few regressed. As Song and Hsu (2011) reminded, the stage a service user reaches might be transitory because recovery is a journey of spiral progress. A longer term intervention is needed before service users can stably progress into a higher stage.

Clearly, the current study provides only preliminary evidence of the applicability and effectiveness of SCM among people in Hong Kong with mental health problems. The study had a number of limitations. First, with the small sample size, it is difficult for the statistical tests to acquire adequate power to detect statistically significant changes. Second, the intervention was implemented for six months and the number of worker-user contacts was on average 5.4. The dosage might be too low to realize the full potential of SCM. Finally, the absence of a control group precludes any definitive conclusions. Despite the limitations, the preliminary findings suggest that a more rigorous design with a control group is warranted. In future applications, it will be useful to extend the period of intervention beyond six months and examine whether hope and autonomy can have rippling effects on the other elements of recovery and mental health status in the long run. Qualitative inquiries to explore service users' responses to SCM will also inform how the approach can be adapted for the local population.

Despite the limitations and modest findings, the current study adds to the existing literature on SCM. In particular, the strengths-based model was originally developed to help people in western cultures which embrace individualism. Chinese people including Hong Kong citizens are characterized by a culture of humility and self-deprecation where it is not easy to help people acknowledge their strengths. However, the experience of this study indicates that with worker's patience this is achievable, and strengths-based practice can be applied in a Hong Kong population.

In conclusion, the current study provides empirical evidence supporting the effectiveness of SCM practice on the recovery of people with mental illness in Hong Kong. With improvement in their autonomy and hope, the individuals in this study have enjoyed improved well-being and a more satisfied life. Though further refinement and testing are vital, adoption of SCM in Hong Kong mental health service is promising.

\section{References}

Barry, K. L., Zeber, J. E., Blow, F. C., \& Valenstein, M. (2003). Effect of strengths model versus assertive community treatment model on participant outcomes and utilization: Two-year follow-up. Psychiatric Rehabilitation Journal, 26(3), 268-277. doi: http://dx.doi.org/10.2975/26.2003.268.277

Becker, D. R., \& Drake, R. E. (2003). A working life for people with severe mental illness. New York: Oxford.

Bond, M. H., Lun, V. M. C., Chan, J., Chan, W. W. Y., \& Wong, D. (2012). Enacting modesty in Chinese culture: The joint contribution of personal characteristics and contextual features. Asian Journal of Social Psychology, 15(1), 14-25. doi: http://dx.doi.org/10.1111/j.1467-839X.2011.01357.x

Deegan, P. (1996, September). Recovery and the conspiracy of hope. Paper presented at the Sixth Annual Mental Health Services Conference of Australia and New Zealand, Brisbane, Australia. Retrieved from https://www.patdeegan.com/patdeegan/lectures/conspiracy-of-hope 
Deegan, P. E. (1988). Recovery: The lived experience of rehabilitation. Psychosocial Rehabilitation Journal, 11(4), 11-19. doi: http://dx.doi.org/10.1037/h0099565

Diener, E., Emmons, R. A., Larsen, R. J., \& Griffin, S. (1985). The Satisfaction with Life Scale. Journal of Personality Assessment, 49, 71-75. doi: http://dx.doi.org/10.1207/s15327752jpa4901_13

Fukui, S., Goscha, R., Rapp, C. A., Mabry, A., Liddy, P., \& Marty, D. (2012). Strengths model case management fidelity scores and client outcomes. Psychiatric Services, 63(7), 708-710. http://dx.doi.org/10.1176/appi.ps.201100373

Goldberg, D. P., \& Williams, P. (1988). A user's guide to the General Health Questionnaire. Windsor, UK: NFER-Nelson.

Hillman, J., \& Ventura, M. (1992). We've had a hundred years of psychotherapy - And the world's getting worse. San Francisco, CA: Harper.

Kisthardt, W. (1993). The impact of the strengths model of case management from the consumer perspective. In M. Harris \& H. Bergman (Eds.), Case management: Theory and practice (pp. 165-182). Washington, DC: American Psychiatric Association.

Kretzmann, J. P., \& McKnight, J. L. (1993). Building communities from the inside out: A path toward finding and mobilizing a community's assets. Evanston, IL: Center for Urban Affairs and Policy Innovations, Northwestern University.

Lee, P. W. H., Lam, T. H., Ong, S. G., Wang, C. M., \& Kleevens, J. W. L. (1985). A modified version of the General Health Questionnaire as a measure of occupational mental health in Hong Kong. Chinese Journal of Psychology, 2, 100-106.

Lee, G. T. H., \& Yiu, M. G. C. (2013). Psychiatric rehabilitation and recovery-Situation of Hong Kong. Hong Kong Journal of Mental Health, 39(1), 4-12.

Liu, A. S. F. (2014). Voices of recovery: What helps in the recovery of people with lived experiences of mental disorder. China Journal of Social Work, 7(1), 26-38. doi: http://dx.doi.org/10.1080/17525098.2014.882785

Macias, C., Farley, O. W., Jackson, R., \& Kinney, R. (1997). Case management in the context of capitation financing: An evaluation of the strengths model. Administration and Policy in Mental Health and Mental Health Services Research, 24(6), 535-543. doi: http://dx.doi.org/10.1007/BF02042831

Macias, C., Kinney, R., Farley, O. W., Jackson, R., \& Vos, B. (1994). The role of case management within a community support system: Partnership with psychosocial rehabilitation. Community Mental Health Journal, 30(4), 323-339. doi: http://dx.doi.org/10.1007/BF02207486

Mak, W. W. S. (2013). Applying a mental illness self-management program effectiveness of Wellness Recovery Action Planning (WRAP) in Hong Kong. Unpublished manuscript, Department of Psychology, The Chinese University of Hong Kong.

Mead, S., \& Copeland, M. E. (2005). What recovery means to us: Consumers' perspectives. In L. Davidson, C. Harding, \& L. Spaniol (Eds.), Recovery from mental illness: Research evidence and implications for practice (pp. 69-81). Boston, MA: Centre for Psychiatric Rehabilitation, Boston University.

Miller, S. D., Hubble, M. A., \& Duncan, B. L. (1996). Handbook of solution-focused brief therapy. San Francisco, CA: Jossey-Bass. 
Modrcin, M., Rapp, C. A., \& Poertner, J. (1988). The evaluation of case management services with the chronically mentally ill. Evaluation and Program Planning, 11(4), 307-314. doi: http://dx.doi.org/10.1016/0149-7189(88)90043-2

Pavot, W., \& Diener, E. D. (1993) Review of the Satisfaction with Life Scale. Psychological Assessment, 5(2), 164-172. doi: http://dx.doi.org/10.1037/10403590.5.2.164

Rapp, C. A. (1994). Theory, principle, and methods of the strengths model of case management. In M. Harris \& H. Bergman (Eds.), Case management: Theory and practice. Washington, DC: American Psychiatric Association.

Rapp, C. A. (1998). The strengths model: Case management with people suffering from severe and persistent mental illness. New York: Oxford.

Rapp, C. A., \& Goscha, R. J. (2004). The principles of effective case management of mental health services. Psychiatric Rehabilitation Journal, 27(4), 319-333. doi: http://dx.doi.org/10.2975/27.2004.319.333

Rapp, C. A., \& Goscha, R. J. (2011). The strengths model: A recovery-oriented approach to mental health services. New York: Oxford University Press.

Rapp, C. A., \& Sullivan, W. P. (2014). The strengths model: Birth to toddlerhood. Advances in Social Work, 15(1), 129-142.

Sachs, J. (2004). Validation of the Satisfaction with Life Scale in a sample of Hong Kong university students. Psychologia: An International Journal of Psychology in the Orient, 46(4), 225-234. doi: http://dx.doi.org/10.2117/psysoc.2003.225

Saleebey, D. (2006). The strengths approach to practice. In D. Saleebey (Ed.), The strengths perspective in social work practice (4th ed., pp. 77-92). Boston, MA: Allyn \& Bacon.

Siegal, H. A., Fisher, J. H., Rapp, R. C., Kelliher, C. W., Wagner, J. H., O’Brien, W. F., \& Cole, P. A. (1996). Enhancing substance abuse treatment with case management: Its impact on employment. Journal of Substance Abuse Treatment, 13(2), 93-98. doi: http://dx.doi.org/10.1016/0740-5472(96)00029-3

Siu, B. W. M., \& Sung, R. W. K. (2013). Promotion and implementation of recoveryoriented practice in a mental hospital in Hong Kong. Hong Kong Journal of Mental Health, 39(1), 13-19.

Smock, S. A., Trepper, T. S., Wetchler, J. L., McCollum, E. E., Ray, R., \& Pierce, K. (2008). Solution-focused group therapy for level I substance abusers. Journal of Marital and Family Therapy, 34(1), 107-120. doi: http://dx.doi.org/10.1111/j.17520606.2008.00056.X

Song, L. Y., \& Hsu, S. T. (2011). The development of the Stages of Recovery Scale for persons with persistent mental illness. Research on Social Work Practice, 21(5), 572581. doi: http://dx.doi.org/10.1177/1049731511402218

Stanard, R. P. (1999). The effect of training in a strengths model of case management on client outcomes in a community mental health center. Community Mental Health Journal, 35(2), 169-179. doi: http://dx.doi.org/10.1023/A:1018724831815

Xie, H. (2013). Strengths-based approach for mental health recovery. Iranian Journal of Psychiatry and Behavioral Sciences, 7(2), 5-10. 
Yeung, L., Ho, K., Yau, S., Tang, J., Wong, S., Tsang, S., Chan, S. K., \& Fung, T. (2013). Peer support worker project in Hong Kong: A local experience and observations. Hong Kong Journal of Mental Health, 39(1), 37-44.

Yip, K. S. (2005). A strengths perspective in working with people with Alzheimer's disease. Dementia: The International Journal of Social Research and Practice, 4(3), 434-441. doi: http://dx.doi.org/10.1177/1471301205055901

Yip, K. S. (2006). A strengths perspective in working with an adolescent with self-cutting behaviors. Child and Adolescent Social Work Journal, 23(2), 134-146. doi: http://dx.doi.org/10.1007/s10560-005-0043-4

\section{Author note}

Address correspondence to: Siu-kau Cheung, PhD, RSWHK, Community College of City University, 88 Tat Chee Avenue, Kowloon Tong, Hong Kong.

Email: scskc@cityu.edu.hk 